\title{
Vitamin E leaves bitter aftertaste
}

Taking a common dose and formulation of vitamin $\mathrm{E}$ as a dietary supplement increases the risk of prostate cancer. This "observation of important public health concern," as described by the authors, was made after extended follow-up of the Selenium and Vitamin E Cancer Prevention Trial (SELECT).

With a lifetime risk of $16 \%$, the incidence of prostate cancer is relatively high in the USA. SELECT was initiated after epidemiological data indicated that selenium and vitamin $\mathrm{E}$ might reduce prostate-cancer risk. About 35,000 healthy men were enrolled between 2001 and 2004 at over 420 study sites across North America and equally allocated to one of four treatment groups: selenium $(20 \mu \mathrm{g} /$ day) plus placebo, vitamin $\mathrm{E}$ (400 IU/day) plus placebo, selenium plus vitamin $\mathrm{E}$, or placebo only. After a median follow-up of 5.5 years, participants stopped taking the supplements when it became clear in 2008 that no reduction in prostate cancer was achieved by any intervention. Concerned by a slightalbeit statistically insignificant-increase in prostate-cancer risk of participants who took vitamin E only, the trial safety monitoring committee decided extended monitoring would be needed to assess a potential harm of vitamin $\mathrm{E}$ supplementation in this population.

Roughly 3 years later, the SELECT investigators obtained additional follow-up data from about 7,600 people in each treatment group. They recorded 521 new cases of prostate cancer among the study population, which increased the total number of primary end points by $30 \%$. Analyzing this extended data set, the team now noted a significant $13 \%$ increase in prostate-cancer risk in the vitamin $\mathrm{E}$ group (hazard ratio [HR] 1.13; 99\% CI 1.004-1.360; $P=0.008)$. As participants who took vitamin $\mathrm{E}$ and selenium had a normal risk (HR 1.05; 99\% CI 0.88-1.25; $P=0.08$ ), selenium might have a positive effect by dampening the increased risk associated with vitamin E. Selenium alone, however, did not seem to affect prostatecancer risk (HR 1.04; 99\% CI 0.87-1.27; $P=0.11$ ), and a previously indicated potential risk of diabetes mellitus with selenium was not confirmed after extended follow-up.

At present, the investigators do not have a biological explanation for their observation, but advocate that vitamin $\mathrm{E}$ and other seemingly innocuous yet biologically active substances should be used with caution. Given that in the USA more than $50 \%$ of individuals over 60 years are taking vitamin E supplements-about a quarter of whom take at least $400 \mathrm{IU} /$ day - these findings might have substantial practical implications and "emphasize the importance of largescale, population-based, randomized trials in accurately assessing the benefits and harms of micronutrients as dietary supplements," the authors write.

Christoph A. Schmitt

Original article Klein et al. Vitamin $\mathrm{E}$ and the risk of prostate cancer. The Selenium and Vitamin E Cancer Trial (SELECT). JAMA 306, 1546-1556 (2011) 